\title{
Dissociable Neural Networks Supporting Metacognition for Memory and Perception
}

\author{
Jane Garrison \\ Department of Psychology, and Behavioural and Clinical Neuroscience Institute, University of Cambridge, CB2 3EB Cambridge, United Kingdom \\ Review of Baird et al.
}

Metacognition in its broadest sense refers both to knowledge about our mental abilities and to the regulatory processes by which we might use that knowledge to control cognition; for example, changing how we perform a task if we feel our current strategy is not optimal (FernandezDuque et al., 2000). While there is debate over the extent to which metacognition relates to existing models of monitoring and cognitive control, the term is generally applied to instances where self-knowledge is either declarative (conscious) or based on nonverbal feelings of uncertainty (Fleming and Dolan, 2012).

This relationship between monitoring and control processes is highlighted in the influential Nelson and Narens (1994) model of metacognition, which suggests a separation between two key levels of regulatory processing: a personal "meta" level that acts both to monitor and control processing at the independent "object" level via feedback (Fig. 1). So an example of metacognitive regulation would be a decision-making process where the choice of an action can be made based on the assessment of potential outcomes (an object-level process), but which can also

Received Dec. 15, 2013; revised Jan. 14, 2014; accepted Jan. 15, 2014.

J.G. is supported by a graduate studentship from the Medical Research Council, UK. I am very grateful to Dr Jon Simons for his helpful comments and advice.

Correspondence should be addressed to Jane Garrison, Department of Psychology, Downing Street, CB2 3EB Cambridge, UK. E-mail: jrg60@cam.ac.uk.

DOI:10.1523/JNEUROSCI.5232-13.2014

Copyright $\odot 2014$ the authors $\quad 0270-6474 / 14 / 342765-03 \$ 15.00 / 0$ be influenced by an awareness of the confidence with which those outcomes have been determined (a meta-level process). Such a theoretical view of metacognition sits neatly with the Norman and Shallice (1986) model of executive function that posits a similar higher-level system that regulates object-level information (in this case, the allocation of attentional resources to differing sensory and cognitive demands) through a process of monitoring and control. As with executive function, a link between the prefrontal cortex (PFC) and metacognition is now well established, with the anterior PFC (aPFC) in particular seen as pivotal in underpinning metacognitive ability (Fleming et al., 2012).

Metacognitive research has focused predominantly on the processes involved in self-knowledge and, in particular, the confidence an individual has regarding their performance on a particular task. In a review of the neural basis of metacognitive ability in memory and decisionmaking, Fleming and Dolan (2012) found that accuracy of such confidence judgments dissociates from underlying task performance and is highly variable across individuals. Baird et al. (2013) use these individual differences in their new study to characterize the brain networks involved in metacognitive knowledge during perceptual and memory tasks.

The perceptual task used by Baird et al. (2013) involved a simple two-choice visual discrimination test involving two screens, each displaying six Gabor gratings (small circular patches of alternating dark and white lines). One of the screens included a single grating that had been oriented marginally away from the vertical, which the participants were asked to identify. In comparison, the memory task required an old/new recognition decision to be made about words that had been included or not included on a previously studied wordlist. For both tasks, participants were asked to make a six-point confidence judgment immediately after each test response. Using signal detection theory (SDT), measures of metacognitive accuracy were then calculated and compared for the two tasks. Despite nearidentical mean levels of underlying accuracy, the confidence scores for the perceptual task were lower than for the memory task, and no significant correlation was found between the metacognitive accuracy for each task and underlying task performance. Together, these findings suggest that participants' ability to judge whether they had got the right answer was unrelated to their performance on the underlying task. Moreover, the accuracy of confidence judgments was also uncorrelated across the memory and perceptual tasks, suggesting a dissociation in metacognitive ability between these two cognitive domains.

To compare the neural networks involved during metacognitive memory and perceptual judgments, resting state functional connectivity analysis was used to relate the individual variability in confidence accuracy to the connectivity between two regions of aPFC previously 


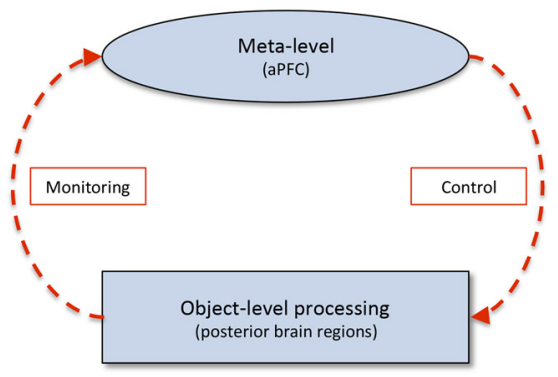

Figure 1. Nelson and Narens model of metacognition (adapted with permission from Nelson and Narens, 1994). The arrows show the flow of metacognitive information and are broken to indicate that object-level processing can occur in the presence or absence of meta-level monitoring and control.

shown to be involved in metacognition: lateral aPFC for perceptual decisions and medial aPFC for mnemonic decisions. Seed regions were defined in right lateral and medial aPFC, their intrinsic connectivity determined, and these connectivity maps used to assess correlations with the metacognitive data for the two tasks. Individual differences in perceptual metacognition were found to correlate with connectivity (as measured by the strength of correlated activity) between the lateral aPFC seed region and clusters in the right dorsal anterior cingulate cortex (ACC), the left putamen, and a cluster that included right putamen, right caudate, and thalamus. For the memory task, the individual difference variability correlated with connectivity between the medial aPFC and two clusters in the right parietal cortex, in the central precuneus, and the intraparietal sulcus (IPS)/inferior parietal lobule (IPL).

There was a key practical difference between the tasks used in the Baird et al. (2013) study. The perceptual task, but not the memory task, was modulated to ensure a consistent accuracy level in underlying task performance, and different SDT measures of metacognitive ability were then used to compare the responses from the two tasks ( $A_{\text {roc }}$ for the perceptual task and $M_{\text {ratio }}$ for the memory task). Although metacognitive accuracy was recalculated for the perceptual task using the $M_{\text {ratio }}$ statistic, it is unclear whether the $M_{\text {ratio }}$ measure of metacognitive ability for the perceptual task was also uncorrelated with underlying task accuracy. However, the $M_{\text {ratio }}$ statistic did confirm the lack of correlation across individuals between perceptual and mnemonic metacognitive accuracy. This finding, together with the differences found in the connectivity networks for the two tasks and the lack of cross-correlation between the two networks (individual differences in perceptual metacognitive ability were uncorrelated with functional connectivity from the medial aPFC and vice versa) supports a dissociation in the neural basis of metacognitive knowledge for mnemonic and perceptual decisions.

Examining the structure of the mnemonic and perceptual metacognitive networks shows that these are largely consistent with the different cognitive demands associated with the two domains. Baird et al. (2013) discuss how the perceptual network can be viewed as a real-time monitor that integrates information over short time-scales, and indeed this network included regions of the striatum and ACC, both linked with a role in monitoring task performance. The present findings are also consistent with recent evidence that accurate perceptual metacognitive judgments of performance depend on the integrity with which lateral aPFC integrates performance monitoring information coded in ACC (Fleming and Dolan, 2012). In contrast, mnemonic metacognitive judgments are different, requiring the appraisal of memory contents or the assessment of relevant memory traces (Nelson and Narens, 1990). The posterior regions of the mnemonic metacognitive network identified by Baird et al. (2013), parietal structures including precuneus and IPS/ IPL, are highly interconnected with the medial temporal lobe, a critical structure in long-term memory encoding and storage. Moreover, a role for the parietal lobe in episodic memory has been suggested based on functional imaging and neuropsychological findings, with recent research indicating a role in the subjective experience of memory (Simons et al., 2010). However, whereas the mnemonic metacognitive network found in the Baird et al. (2013) study included right parietal clusters, indications of parietal involvement in episodic retrieval success are more generally associated with left hemispheric activations (Wagner et al., 2005). Similarly, an investigation into the subjective sense of memory strength found that confidence in relating the gist of a scene correlated with left rather than right hemisphere lateral parietal activations (Qin et al., 2011). The significance of this discrepancy regarding laterality is unclear.

How do these findings inform our understanding of the neural basis of metacognition? The Baird et al. (2013) study identified brain regions on the basis of correlations with individual differences in metacognitive ability, an approach that is less likely to detect similarities in the networks. As such, the authors make clear that a complete dissociation between the mnemonic and perceptual networks cannot be assumed, as there may be common elements underpinning metacognitive processes. Despite this caveat, the extent of dissociation detected in the neural networks supporting metacognitive processes for memory and perception is most consistent with a domain-specific version of the Nelson and Narens model that assumes separable metacognitive networks associated with different cognitive abilities (Nelson and Narens, 1994).

The networks revealed by Baird et al. (2013) also reinforce a view in which the meta-level of processing in the Nelson and Narens model (Nelson and Narens, 1994) maps to the anterior PFC and the objectlevel maps to posterior brain regions, a view consistent with Shimamura's (2000) dynamic filtering theory, which suggests that the PFC monitors and regulates posterior regions through a filtering or gating mechanism. While it is easy to understand the differences in the posterior networks given the variable task demands, it is notable that the networks are also connected to distinct regions of the aPFC, suggesting that both meta-level and object-level processes are domain-specific. Further evidence for a separation of metacognitive function within the PFC comes from earlier work by Fleming and Dolan (2012), who reviewed evidence for a dissociation between retrospective and prospective confidence judgments (those made before and after the relevant task response), which are supported by anterior and dorsal regions of lateral PFC and by medial PFC, respectively. Such a dissociation in prefrontal-cortical activity may simply be organizational given the different connectivity to posterior object-level networks, or there may be inherent differences in the meta-level representational requirements relating to the different domains. However, such tight functional specialization of monitoring functions in the PFC is consistent with previous findings suggesting fine-grained segregation of function between adjacent but dissociable regions of medial aPFC (Gilbert et al., 2010).

While the Baird et al. (2013) study provides a considerable advance in our understanding of metacognition at a neural organization level, key questions remain of how metacognitive self-knowledge comes about and how mistakes are made. Inherent curiosity to drive research forward in these areas seems assured, given 
the importance of metacognition to our sense of self and identity.

\section{References}

Baird B, Smallwood J, Gorgolewski KJ, Margulies DS (2013) Medial and lateral networks in anterior prefrontal cortex support metacognitive ability for memory and perception. J Neurosci 33:16657-16665. CrossRef Medline

Fernandez-Duque D, Baird JA, Posner MI (2000) Executive attention and metacognitive regulation. Conscious Cogn 9:288-307. CrossRef Medline

Fleming SM, Dolan RJ (2012) The neural basis of metacognitive ability. Philos Trans R Soc Lond B Biol Sci 367:1338-1349. CrossRef Medline
Fleming SM, Huijgen J, Dolan RJ (2012) Prefrontal contributions to metacognition in perceptual decision making. J Neurosci 32: 6117-6125. CrossRef Medline

Gilbert SJ, Henson RN, Simons JS (2010) The scale of functional specialisation within human prefrontal cortex. J Neurosci 30:12331237. CrossRef Medline

Nelson T, Narens L (1994) Why investigate metacognition. In: Metacognition: knowing about knowing (Metcalfe J, Shimamura AP, eds.), pp 1-25. Cambridge MA: MIT.

Norman D, Shallice T (1986) Attention to action: willed and automatic control of behavior. In: Consciousness and self-regulation IV (Davidson R, Schwartz R, Shapiro D, eds.), pp 1-18. New York: Plenum.

Qin S, van Marle HJ, Hermans EJ, Fernández
G (2011) Subjective sense of memory strength and the objective amount of information accurately remembered are related to distinct neural correlates at encoding. J Neurosci 31:8920-8927. CrossRef Medline

Shimamura AP (2000) Toward a cognitive neuroscience of metacognition. Conscious Cogn 9:313-323. CrossRef Medline

Simons JS, Peers PV, Mazuz YS, Berryhill ME, Olson IR (2010) Dissociation between memory accuracy and memory confidence following bilateral parietal lesions. Cereb Cortex 20:479-485. CrossRef Medline

Wagner AD, Shannon BJ, Kahn I, Buckner RL (2005) Parietal lobe contributions to episodic memory retrieval. Trends Cogn Sci 9:445453. CrossRef Medline 